\title{
Mechanisms and factors controlling hygroscopic properties of black carbon (BC) from different carbon sources under different humidity conditions
}

Minli Wang, ${ }^{1}$ Yiqun Chen, ${ }^{1}$ Heyun Fu, ${ }^{1}$ Xiaolei Qu, ${ }^{1}$ Bengangl Li, ${ }^{2}$ Shu Tao, ${ }^{2}$ and Dongqiang $\underline{\mathrm{Zhu}}^{2}$

${ }^{1}$ School of the Environment, Nanjing University, Jiangsu 210046, China

${ }^{2}$ School of Urban and Environmental Sciences, Peking University, Beijing 100871, China

The hygroscopic behavior of carbonaceous aerosols has a significant impact on global and regional climate change. However, the mechanism and factors controlling the hygroscopicity of black carbon (BC) from different carbon sources are not well understood. Here, we systematically measured the equilibrium and kinetics of water uptake by 15 different BC (10 herb-derived $\mathrm{BC}, 2$ wood-derived $\mathrm{BC}$, and 3 soot) using gravimetric water vapor sorption method combined with in-situ diffuse reflectance infrared Fourier transform spectroscopy (DRIFTS). In the gravimetric analysis, the sorption/desorption equilibrium isotherms were measured under continuous-stepwise water vapor pressure conditions, while the kinetics was measured at a variety of humidity levels obtained by different saturated aqueous salt solutions. The equilibrium water uptake of the $\mathrm{BC}$ pool at high relative humidity $(>80 \%)$ positively correlated to the dissolved mineral content $(0.01-13.0 \mathrm{wt} \%)\left(R^{2}=0.96, P=0.0001\right)$ as well as the content of the thermogravimetrically analyzed organic carbon $\left(\mathrm{OC}_{\mathrm{TGA}}, 4.48-15.25 \mathrm{wt} \%\right)$ $\left(R^{2}=0.82, P=0.0001\right)$ and the alkali-extracted organic carbon $\left(\mathrm{OC}_{\mathrm{AE}}, 0.14-8.39 \mathrm{wt} \%\right)\left(R^{2}=\right.$ $0.80, P=0.0001)$. By contrast, no positive correlation was obtained with the content of total organic carbon or elemental carbon. Among the soluble ionic constituents, chloride, nitrate, and ammonium were each correlated with the equilibrium water uptake at high relative humidity. Compared with the herbal BC and soot, the woody BC had much lower equilibrium water uptake, especially at high relative humidity, likely due to the very low dissolved material content and OC content. The DRIFTS analysis provided generally consistent results at low relative humidity. The results indicated that the equilibrium water uptake by $\mathrm{BC}$ was mainly controlled by the hygroscopic constituents of $\mathrm{OC}$ and dissolved mineral. The kinetic water uptake by the $\mathrm{BC}$ could be well described by the pseudo-second order kinetics model. The calculated rate constant $\left(k_{2}\right)$ positively correlated to the contents $\mathrm{OC}_{\mathrm{TGA}}\left(R^{2}=0.47, P=\right.$ $0.0046), \mathrm{OC}_{\mathrm{AE}}\left(R^{2}=0.44, P=0.0070\right)$, and $\mathrm{NH}_{4}{ }^{+}-, \mathrm{Cl}^{-}$-, and $\mathrm{NO}_{3}^{-}$-salts $\quad\left(R^{2}=053-0.77, P=\right.$ $0.0001-0.0021)$ under low humidity conditions, but to the total porosity only under high humidity conditions $\left(R^{2}=0.82, P=0.0001\right)$. The fast water uptake kinetics under low humidity conditions was attributed to the binding to high affinity, active sites (OC and salts), whereas the slow water uptake kinetics under high humidity conditions was attributed to pore-filling/condensation of water molecules within the micro- and mesopores of BC. This study highlights that the hygroscopic properties of $\mathrm{BC}$ rely on compositional and structural properties of $\mathrm{BC}$ as well as humidity conditions.

Keywords: hygroscopicity; black carbon; organic carbon; minerals; humidity 